\title{
Pulsed electromagnetic fields inhibit osteoclast differentiation in RAW264.7 macrophages via suppression of the protein kinase $B /$ mammalian target of rapamycin signaling pathway
}

\author{
YUTIAN LEI ${ }^{1}$, JINYU SU ${ }^{2}$, HAIXIA XU ${ }^{1}$, QIANG YU ${ }^{1}$, MING ZHAO $^{2}$ and JING TIAN ${ }^{1}$ \\ ${ }^{1}$ Department of Orthopaedics, Zhujiang Hospital, Southern Medical University, Guangzhou, Guangdong 510280; \\ ${ }^{2}$ Department of Pathophysiology, Key Laboratory for Shock and Microcirculation Research of Guangdong, \\ Southern Medical University, Guangzhou, Guangdong 510515, P.R. China
}

Received November 12, 2017; Accepted April 17, 2018

DOI: $10.3892 / \mathrm{mmr} .2018 .8999$

\begin{abstract}
When bone resorption, aided by the activity of osteoclasts, exceeds bone formation induced by osteoblasts, bone metabolism loses equilibration, which results in the development of bone diseases, including osteoporosis. Pulsed electromagnetic fields (PEMFs) are known to be involved in various biological processes, including cell proliferation, differentiation and apoptosis. However, the exact mechanism of action of osteoclasts remains poorly understood. In the present study, the effects of PEMFs on osteoclast differentiation and associated signaling pathways were systematically investigated in RAW264.7 macrophages. RAW264.7 cells were induced by receptor activator of nuclear factor- $\mathrm{\kappa B}$ ligand (RANKL) to obtain osteoclasts in vitro. The results of the present study demonstrated that PEMF exposure decreased osteoclast formation, limited tartrate-resistant acid phosphatase activity, contracted bone resorption area and inhibited osteoclastic specific gene and protein expression. Furthermore, western blot analysis indicated that PEMFs distinctly abolished the upregulation of phosphorylated-protein kinase B (Akt), -mammalian target of rapamycin (mTOR) and -ribosome S6 protein kinase (p70S6K) induced by RANKL, which was consistent with the effects of pharmacological inhibitor perifosine and rapamycin. Therefore, the present study suggested that PEMFs reduced osteoclast formation from RAW264.7 macrophages via inhibition of the Akt/mTOR signaling pathway. These findings provided novel insight into
\end{abstract}

Correspondence to: Dr Jing Tian, Department of Orthopaedics, Zhujiang Hospital, Southern Medical University, 253 Industrial Avenue, Haizhu, Guangzhou, Guangdong 510280, P.R. China E-mail: tianjing_ortho@163.com

Key words: pulsed electromagnetic fields, receptor activator of nuclear factor- $\mathrm{kB}$ ligand, osteoclast differentiation, protein kinase $\mathrm{B}$, mammalian target of rapamycin, osteoporosis the mechanisms through which PEMFs suppress osteoclast differentiation.

\section{Introduction}

Osteoporosis is a common clinical disease exerting tremendous emotional, economic and social repercussions on patients and their families (1). Traditional pharmacological agents either promoting bone formation (e.g., parathyroid hormone) or inhibiting bone resorption (e.g., calcitonin, estrogen and bisphosphonate) may contribute to the prevention and reversal of osteoporosis to a certain extent; however, undesirable side effects, including metrorrhagia, esophagitis and mammary cancer, may occur simultaneously $(2,3)$. Despite extensive research in experimental studies and preclinical trials of other novel pharmacological agents (e.g., denosumab, odanacatib and saracatinib), the potential mechanisms and possible side effects are not fully understood (4-6). Bonnick et al (7) found that odanacatib is a selective cathepsin $\mathrm{K}$ inhibitor being developed for the treatment of osteoporosis, but it increased the risk of atrial fibrillation and stroke. Furthermore, certain drugs are too expensive for patients to afford due to the long courses of drug treatment required.

Since the first application of pulsed electromagnetic fields (PEMFs) in accelerating clinical bone fracture healing in $1974(8,9)$, the biological effects of PEMFs have gained considerable attention in orthopaedic research. Over the past four decades, studies regarding electromagnetic fields on different cells and animals have been extensively described. Accumulating evidence has revealed that PEMF stimulation potently promotes osteogenesis and enhances bone mineralization both in vivo and in vitro (10-12). Other studies have indicated that electromagnetic field treatment exerts protective effects on human bone marrow mesenchymal stem cells (13). Kang et al (14) suggested that the osteogenic differentiation of adipose-derived stem cells and bone regeneration is accelerated by PEMF stimulation. He et al (15) demonstrated that PEMFs significantly reduce the number of osteoclast-like cells in the culture with macrophage colony stimulating factor 
(M-CSF) and receptor activator of nuclear factor- $\mathrm{kB}$ ligand (RANKL), which indicates the potential role of PEMFs in osteoporosis. Despite the considerable beneficial effects of PEMF on osteoporosis $(16,17)$, the underlying mechanism remains to be fully elucidated, which may impose restrictions for the clinical application of PEMFs.

The pivotal role of osteoclasts in bone defects and osteoporosis have resulted in their becoming a key therapeutic target in osteoporosis (18). Previous studies have investigated the effects of PEMFs on osteoblasts and osteoclasts. The results have demonstrated that PEMFs inhibit the differentiation of osteoclasts and facilitate the formation of osteoblasts, the underlying mechanisms of which are different $(19,20)$. The promotion of osteoblast differentiation by PEMFs is primarily focused on the bone morphogenetic protein and the Wnt/ $\beta$-catenin signaling pathways, the activation of which facilitates osteoblast differentiation, and improves bone microstructure and strength $(21,22)$. Various studies have suggested that PEMF may suppress osteoclast differentiation by regulating certain pathways in the RANK/RANKL/osteoprotegerin (OPG) signaling system (23), of which the protein kinase B (Akt)/mammalian target of rapamycin (mTOR) signaling pathway may be key (24). The present study aimed to elucidate the effects of PEMFs on RANKL-induced osteoclast differentiation from RAW264.7 macrophages and to explore the potential mechanisms involving the intracellular Akt/mTOR signaling pathway during this process.

\section{Materials and methods}

Cell culture and reagents. RAW264.7 cells were purchased from the American Type Culture Collection (Manassas, VA, USA). Cells were cultured in Dulbecco's modified Eagle's medium (DMEM; Thermo Fisher Scientific, Inc., Waltham, MA, USA; $4.500 \mathrm{mg} / \mathrm{l} \mathrm{D}$-glucose) supplemented with $10 \%$ fetal bovine serum (FBS; Gibco; Thermo Fisher Scientific, Inc.) for maintaining cell growth or in $\alpha$-minimum essential medium ( $\alpha$-MEM; Gibco; Thermo Fisher Scientific, Inc., $1.000 \mathrm{mg} / \mathrm{l} \mathrm{D}$-glucose) supplemented with 10\% FBS (Gibco; Thermo Fisher Scientific Inc.) for inducing cell differentiation, at $37^{\circ} \mathrm{C}$ in an environment containing $5 \% \mathrm{CO}_{2}$. Recombinant mouse RANKL was obtained from R\&D Systems (Minneapolis, MN, USA). Cell Counting Kit-8 (CCK-8) was purchased from Dojindo Molecular Technologies, Inc. (Kumamoto, Japan) and a tartrate-resistant acid phosphatase (TRAP) staining kit was purchased from Sigma-Aldrich; Merck KGaA (Darmstadt, Germany). Bovine bone slices were obtained from Third Military Medical University (Chongqing, China). cDNA Synthesis kits and All-in-One qPCR Mix were purchased from GeneCopoeia (Rockville, MD, USA). Antibodies against Akt, mTOR, ribosome $\mathrm{S} 6$ protein kinase (p70S6K), phosphorylated (p)-Akt, p-mTOR, p-p70S6K, nuclear factor of activated T-cells 1 (NFATc1), GAPDH and anti-rabbit horseradish peroxidase-conjugated antibodies were purchased from Cell Signaling Technology, Inc. (Danvers, MA, USA). Antibodies against matrix metallopeptidase (MMP)-9, TRAP and cathepsin K (CTSK) were purchased from Proteintech (Rocky Hill, NJ, USA). Inhibitors perifosine and rapamycin were purchased from Selleck Chemicals (Houston, TX, USA).
PEMF stimulation. To explore the effects of PEMFs on osteoclast differentiation, an electromagnetic field device was used, as previously described (25). Based on previous studies and the preliminary work of the present study, the appropriate parameters of PEMFs with $50 \mathrm{~Hz}$ and $1 \mathrm{mT}$ were selected. Briefly, RAW264.7 macrophages were exposed to PEMFs in a system formed by Helmholtz coils (inner diameter of $\sim 30 \mathrm{~cm}$ ) that oriented to produce a sinusoidal PEMF with $50 \mathrm{~Hz}$ and $1 \mathrm{mT}$. All studies were conducted in a humidified incubator at $37^{\circ} \mathrm{C}$ under $5 \% \mathrm{CO}_{2}$ for $4 \mathrm{~h}$ per day. The control group was cultured in a separate incubator under the same conditions without exposure to PEMFs. Medium was changed every 2 days.

CCK-8 assay for cell viability. Cell viability was tested using a CCK-8 assay. RAW264.7 macrophages were seeded at a density of $2 \times 10^{3}$ cells/well onto a 96 -well culture plate in DMEM. Following the overnight incubation for attachment to the wall, the culture medium was changed to $\alpha$-MEM and cells were cultured further in a humidified incubator with RANKL $(50 \mathrm{ng} / \mathrm{ml})$, RANKL (50 ng/ml) + PEMF, perifosine $(2.5 \mu \mathrm{M})$, rapamycin $(1 \mu \mathrm{M})$ and PEMFs for 4 days. A CCK- 8 assay was carried out every $24 \mathrm{~h}(24,48,72$ and $96 \mathrm{~h})$ according to the manufacturer's protocol. CCK-8 reagent was added to each well, followed by incubation for $4 \mathrm{~h}$, prior to absorbance being measured at a wavelength of $450 \mathrm{~nm}$ using a microplate reader.

TRAP staining and activity. RAW264.7 macrophages were seeded at a density of $1.5 \times 10^{4}$ cells/well onto a 24 -well culture plate in DMEM overnight. Then, the medium was changed to $\alpha$-MEM with the addition of RANKL $(50 \mathrm{ng} / \mathrm{ml})$ or processed with RANKL + PEMF (50 Hz, $1 \mathrm{mT}$ ) for 4 days to induce osteoclast differentiation. Then, cells were fixed with $3.7 \%$ formaldehyde for $30 \mathrm{sec}$ and were stained with TRAP staining solution ( $45 \mathrm{ml}$ deionized water, $0.5 \mathrm{ml}$ Fast Garnet GBC Base solution, $0.5 \mathrm{ml}$ sodium nitrite solution, $0.5 \mathrm{ml}$ naphthol AS-BI phosphate solution, $2 \mathrm{ml}$ acetate solution and $1 \mathrm{ml}$ tartrate solution) according to the manufacturer's protocol. TRAP-positive osteoclasts containing three or more nuclei were counted in 12 wells/plate. This counting was repeated five times. TRAP activity was measured from osteoclast culture supernatants using a TRAP Staining kit. In brief, supernatants (30 $\mu 1$ per well) were incubated with the chromogenic substrates $(170 \mu \mathrm{l})$ in a tartrate-containing buffer for $3 \mathrm{~h}$ at $37^{\circ} \mathrm{C}$. Then, absorbance was measured to determine TRAP activities at a wavelength of $540 \mathrm{~nm}(26)$.

Bone resorption assay. Cells were seeded at a density of $1.5 \times 10^{4}$ cells/well onto a 48 -well plate covered with bovine bone slices. RAW264.7 macrophages were then induced with RANKL (50 or $100 \mathrm{ng} / \mathrm{ml}$ ) for 8 days with or without PEMF exposure. Following 8 days of culture, the bovine bone slices were washed with $5 \%$ sodium hypochlorite solution and toluidine blue staining was performed. To quantify the osteoclastic bone resorption, the resorbed pit areas were confirmed under a light microscope and identified by Image pro-plus (version 6.0).

Reverse transcription-quantitative polymerase chain reaction (RT- $q P C R$ ) analysis. Cells were washed with DPBS, and total RNA was extracted using $1 \mathrm{ml}$ TRIzol reagent. Samples 
homogenized in TRIzol reagent were then extracted using $0.2 \mathrm{ml}$ chloroform. Following centrifugation at 10,000 rpm at $4^{\circ} \mathrm{C}$ for $15 \mathrm{~min}$, the supernatant containing RNA was transferred into a new vial and RNA was precipitated by adding $500 \mu 1$ isopropanol. The supernatant was discarded following incubation for $10 \mathrm{~min}$ and centrifugation at 10,000 rpm for $10 \mathrm{~min}$. The pellet was washed with $1 \mathrm{ml} 75 \%$ ethanol and centrifuged for $5 \mathrm{~min}$ at 7,000 rpm. The supernatant was then discarded and the pellet was dried. After adding $30 \mu \mathrm{l}$ diethylpyrocarbonate (DEPC)-treated water, the pellet was dissolved at $85^{\circ} \mathrm{C}$ for $10 \mathrm{~min}$. Total RNA $(1 \mu \mathrm{g})$ was then reverse transcribed with the cDNA synthesis kit to obtain cDNA, using the following temperature protocol: $37^{\circ} \mathrm{C}$ for $60 \mathrm{~min}$, followed by $98^{\circ} \mathrm{C}$ for $5 \mathrm{~min}$. qPCR was conducted in an ABI StepOnePlus Real-time PCR system (Applied Biosystems; Thermo Fisher Scientific, Inc.) using ALL-in-One qPCR Mix. The thermocycling conditions were as follows: $95^{\circ} \mathrm{C}$ for $10 \mathrm{~min}$ followed by $95^{\circ} \mathrm{C}$ for $10 \mathrm{sec}$, then 45 cycles of $60^{\circ} \mathrm{C}$ for $20 \mathrm{sec}$ and $72^{\circ} \mathrm{C}$ for $15 \mathrm{sec}$. The relative expression of genes was calculated using the $2^{-\Delta \Delta \mathrm{Cq}}$ method and all results were normalized to GAPDH (27). The sequences of the forward and reverse primers were as follows: GAPDH, ACCACAGTCCAT GCCATCAC and TCCACCACCCTGTTGCTGTA; NFATc1, GGTAACTCTGTCTTTCTAACCTTAAGCTC and GTG ATGACCCCAGCATGCACCAGTCACAG; MMP-9, CGC TCATGTACCCGCTGTAT and TGTCTGCCGGACTCA AAGAC; TRAP, CTGGAGTGCACGATGCCAGCGACA and TCCGTGCTCGGCGATGGACCAGA; and CTSK, AGG CAGCTAAATGCAGAGGGTACA and ATGCCGCAGGCG TTGTTCTTATTC.

Western blotting. Cells were treated for the indicated time with various treatments. Then, western blotting was performed according to standard procedures. Cells were rinsed with PBS and harvested in radioimmunoprecipitation assay buffer containing protease and phosphatase inhibitors. Following incubation on ice for $30 \mathrm{~min}$, the cell lysates were centrifuged at $12,000 \mathrm{rpm}$ for $20 \mathrm{~min}$ and protein precipitations were collected. Proteins (20 ug) were separated on SDS-PAGE (8-12\% gels) and devolved to nitrocellulose membranes. The membranes were blocked with $5 \%$ bovine serum albumin in TBST containing $0.1 \%$ Tween-20 at room temperature for an hour and probed successively with rabbit primary antibodies against the following: MMP-9 (1:1,000), TRAP (1:1,000), CTSK (1:500), NFATc1 (1:1,000), Akt $(1: 1,000)$, p-Akt $(1: 2,000)$, mTOR $(1: 1,000)$, p-mTOR $(1: 1,000)$, p70S6K $(1: 1,000), \mathrm{p}-\mathrm{p} 70 \mathrm{~S} 6 \mathrm{~K}(1: 1,000), \beta$-actin $(1: 1,000)$ and GAPDH $(1: 2,000)$ primary antibodies overnight at $4^{\circ} \mathrm{C}$. Horseradish peroxidase-conjugated goat anti-rabbit $\mathrm{IgG}$ antibodies were used as secondary antibodies at room temperature for an hour. The signals were detected by exposure in an enhanced chemiluminscence system system and then analyzed using ImageJ software (version 1.46).

Statistical analysis.. All data are expressed as the mean \pm standard deviation. Statistical analyses were performed using Student's t-tests or one-way analysis of variance, followed by Student-Newman-Keuls post hoc test, using SPSS software, version 20.0 (IBM Corp., Armonk, NY, USA). P<0.05 was considered to indicate a statistically significant difference.

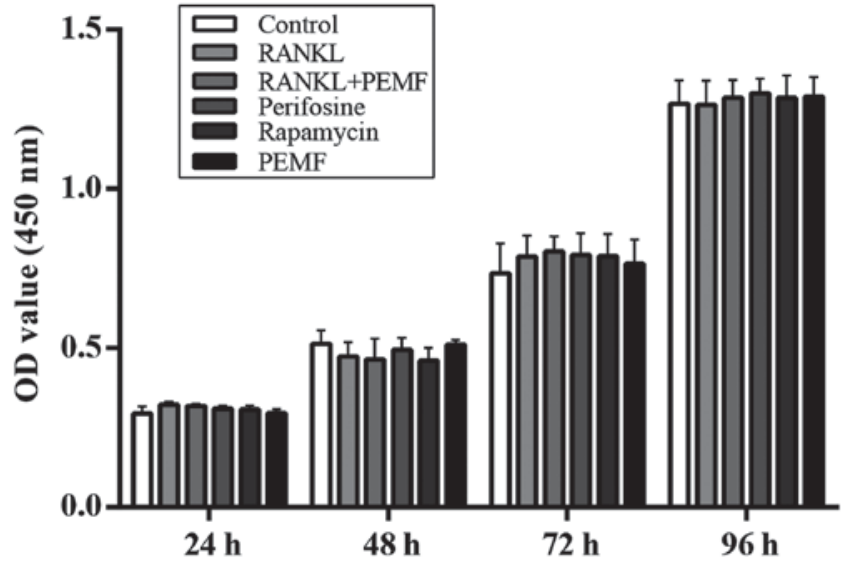

Figure 1. Effect of pulsed electromagnetic fields on cell viability. RAW264.7 macrophages were treated with different stimuli for 4 days. Cell viability was determined using the Cell Counting Kit- 8 assay every 24 h. Data are presented as the mean \pm standard deviation of three independent experiments.

\section{Results}

Effect of PEMF on cell viability. To investigate whether RANKL, PEMF or pharmacological inhibitors influenced cell viability, a CCK-8 assay was performed. RAW264.7 macrophages were cultured with different stimuli for 4 days. The $450 \mathrm{~nm}$ absorbance was detected every $24 \mathrm{~h}$ to assess cell viability post-incubation with the CCK-8 reagent. As presented in Fig. 1, over the four day period, there was no significant difference observed among the different groups, with respect to cell viability, suggesting that RANKL, PEMF and pharmacological inhibitors had no cytotoxic effects on osteoclast precursor cells.

Effect of PEMF on osteoclast formation and TRAP activity. TRAP enzyme is highly expressed and secreted in mature osteoclasts, and functions as a secure indicator for osteoclast formation (28). To explore the influence of PEMF on osteoclast formation, TRAP staining and measurement of TRAP activity was conducted. Osteoclasts containing three or more nuclei were categorized as TRAP-positive cells. When treated with RANKL, the number of giant osteoclasts containing multiple nuclei was markedly increased compared with the control (Fig. 2A). However, the number of multinuclear osteoclasts induced by RANKL was significantly decreased by PEMF application (Fig. 2B). Results of the TRAP activity assay also demonstrated that RANKL treatment resulted in enhancement of TRAP activity, while this facilitating effect was attenuated by PEMF application (Fig. 2C). The suppression of multinucleated osteoclast formation and TRAP activity during osteoclast differentiation suggested that PEMF decreased osteoclastogenesis via osteoclast precursor (RAW264.7 macrophages) fusion inhibition.

Effect of PEMF on osteoclastic bone resorption in vitro. Mature osteoclasts function to absorb bone matrix, resulting in an irregular surface of the matrix (29). The present study investigated whether PEMF could inhibit osteoclastic bone resorption in vitro (Fig. 3). RANKL-induced osteoclasts resulted in resorption pits on bovine bone slices (Fig. 3A). 
A

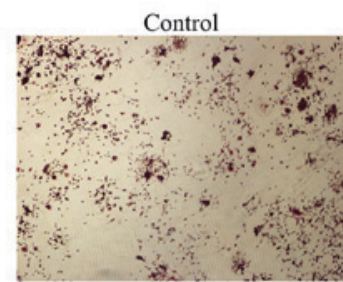

B

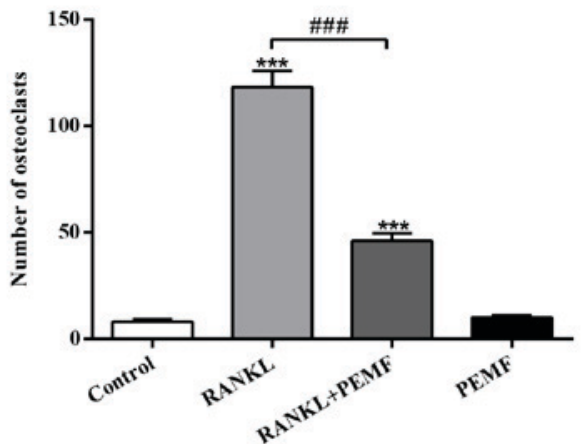

RANKL

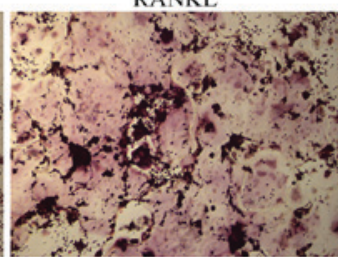

.
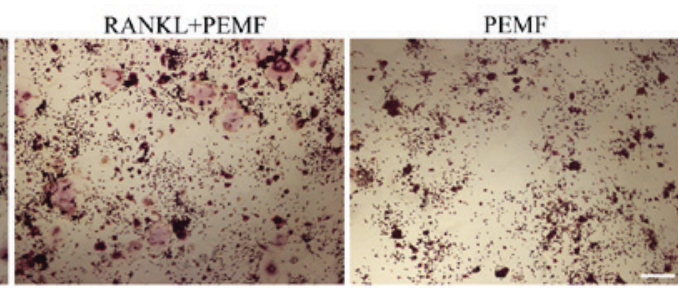

C

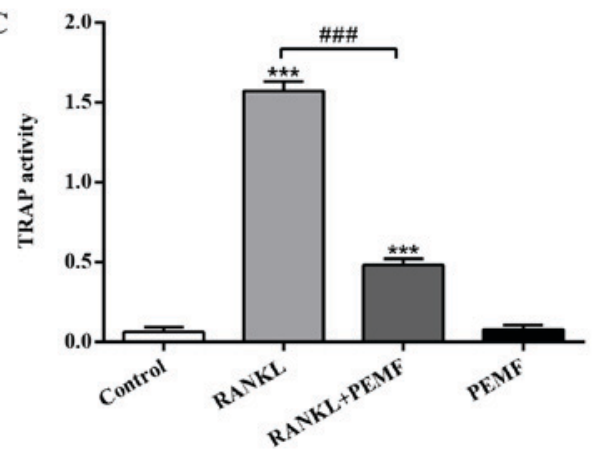

Figure 2. PEMF inhibits RANKL-induced osteoclast formation. (A) RAW264.7 macrophages were treated with or without RANKL (50 ng/ml) in the presence or absence of PEMF exposure for 4 days. Cells were stained for TRAP. Original magnification, $\mathrm{x} 4$. Scar bar=50 $\mu \mathrm{m}$. (B) TRAP-positive multinucleated cells (nuclei $\geq 3$ ) were counted manually and using a nuclei-counter plug-in for ImageJ. (C) TRAP activity was quantitatively measured. Data are presented as the mean \pm standard deviation of three independent experiments. ${ }^{* * *} \mathrm{P}<0.001 \mathrm{vs}$. control; ${ }^{\# \# \#} \mathrm{P}<0.001$ as indicated, based on Student's t-tests and one-way analysis of variance. PEMF, pulsed electromagnetic fields; TRAP, tartrate-resistant acid phosphatase; RANKL, receptor activator of nuclear factor- $\mathrm{kB}$ ligand.

$\mathbf{A}$

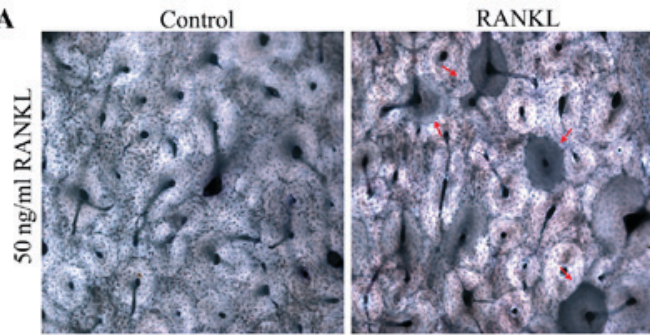

B

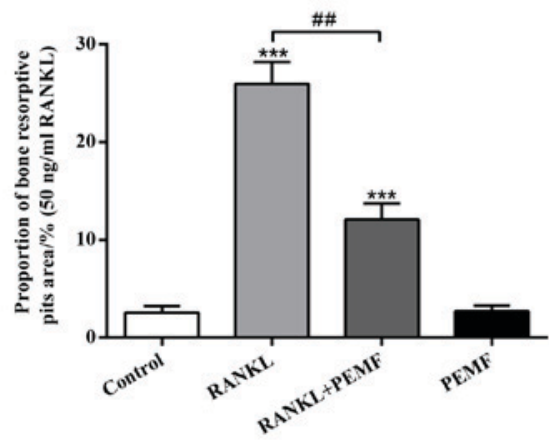

C
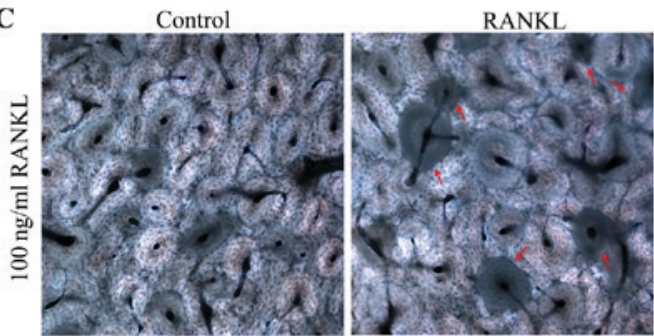

RANKL+PEMF

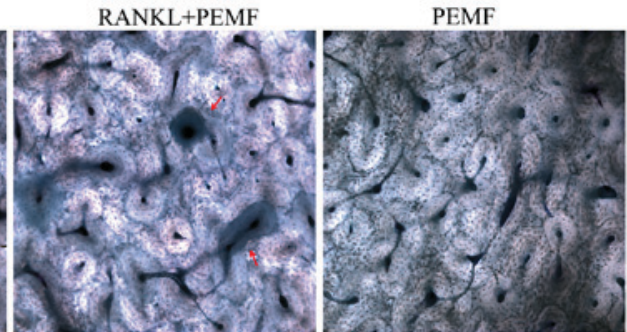

D

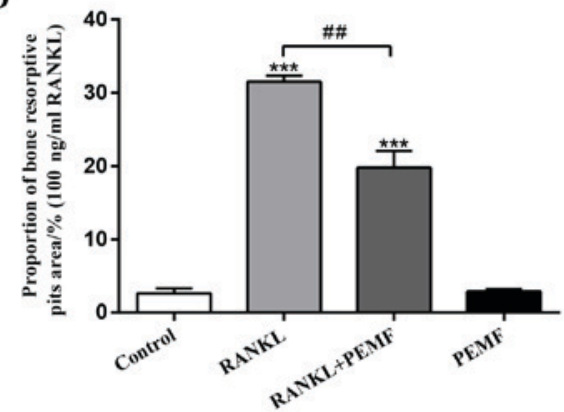

RANKL+PEMF

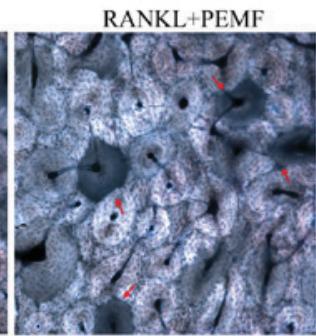

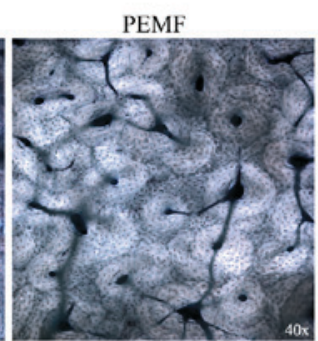

Figure 3. (A-D) PEMF inhibits RANKL-induced bone resorption. (A and C) RAW264.7 macrophages were cultured on bone slices with or without RANKL $(50$ or $100 \mathrm{ng} / \mathrm{ml})$ in the presence or absence of PEMF exposure for 8 days. Osteoclasts were removed and resorption pits on bone slices were then stained with toluidine blue. Original magnification, x10. Scar bar=100 $\mu \mathrm{m}$. The red arrows show resorption pits. (B and D) Proportion of bone resorptive pit areas formed by osteoclasts were analyzed and processed using the Image pro-plus system. Data are presented as the mean \pm standard deviation of three independent experiments. ${ }^{* * *} \mathrm{P}<0.001$ vs. control; ${ }^{\# \#} \mathrm{P}<0.01$ as indicated, based on Student's t-tests and one-way analysis of variance. PEMF, pulsed electromagnetic fields; RANKL, receptor activator of nuclear factor- $\mathrm{kB}$ ligand. 
A

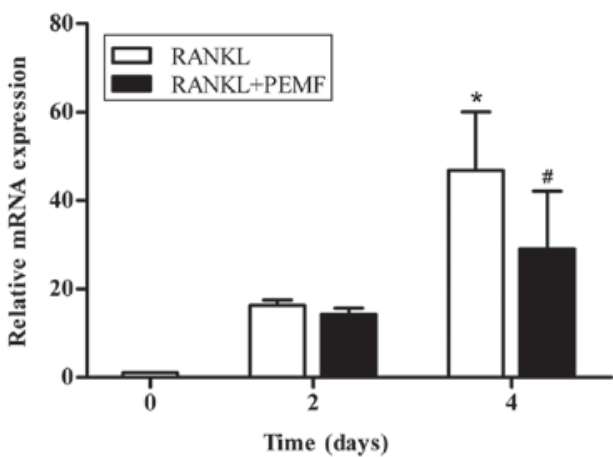

C

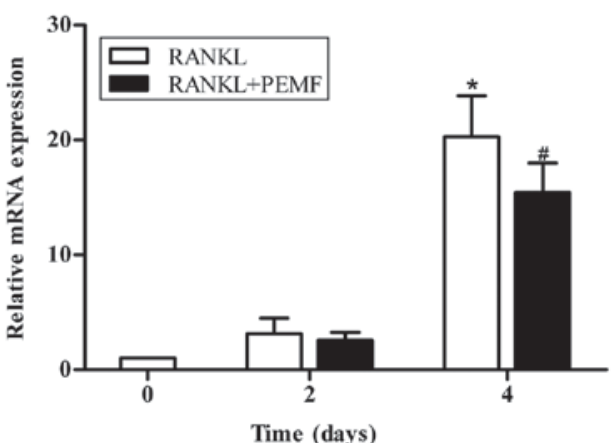

$\mathbf{E}$

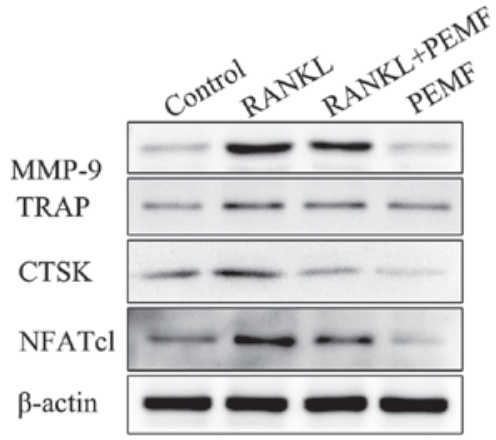

B

TRAP

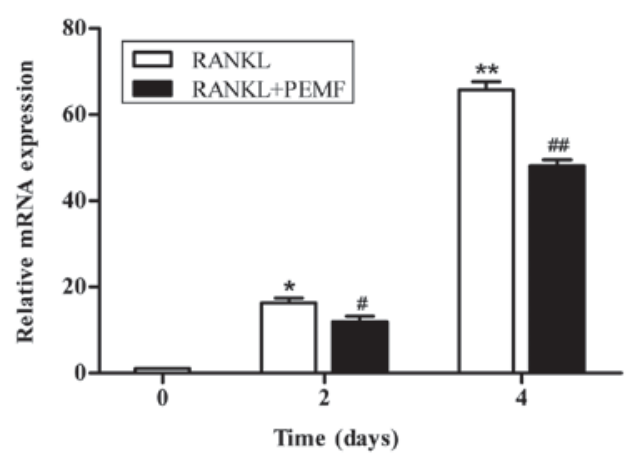

D
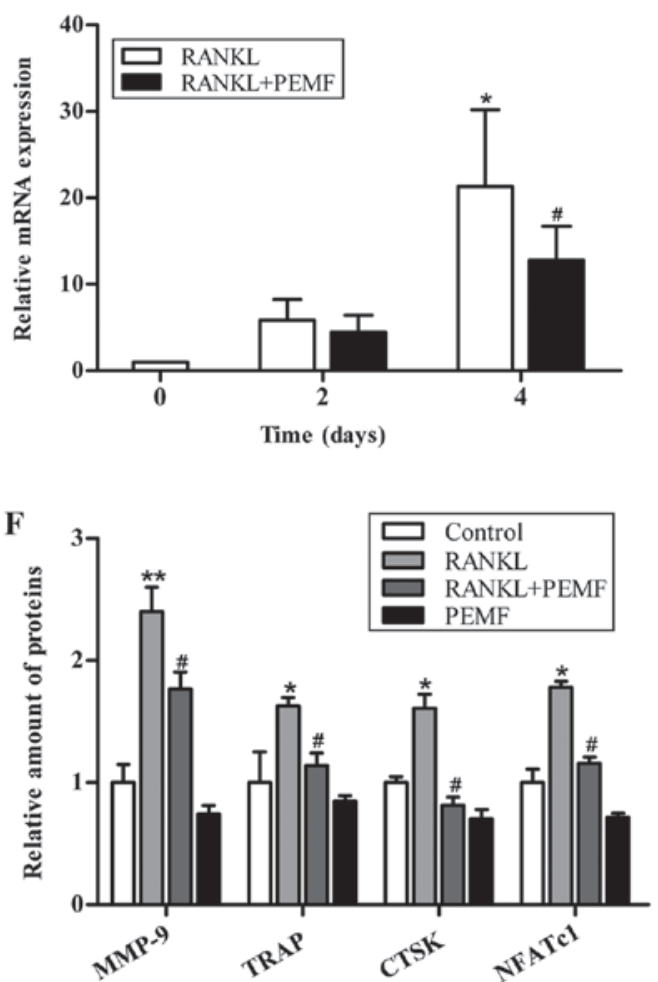

Figure 4. PEMF suppresses RANKL-induced osteoclastic specific gene and protein expression. Cells were exposed to RANKL (50 ng/ml) alone or in combination with PEMF exposure for 2 or 4 days. Gene expression levels of osteoclastic markers (A) MMP-9, (B) TRAP, (C) CTSK and (D) NFATc1 were determined by reverse transcription-quantitative polymerase chain reaction. GAPDH served as a loading control. (E) On day 4, lysates were immunoblotted with MMP-9, TRAP, CTSK and NFATc1 antibodies. $\beta$-actin served as a loading control. (F) Relative protein expression levels were measured using ImageJ software. Data are presented as the mean \pm standard deviation of three independent experiments. ${ }^{*} \mathrm{P}<0.05,{ }^{* *} \mathrm{P}<0.01$ vs. control group; ${ }^{\#} \mathrm{P}<0.05,{ }^{\# \#} \mathrm{P}<0.01$ vs. RANKL group, based on Student's t-tests and one-way analysis of variance. PEMF, pulsed electromagnetic fields; TRAP, tartrate-resistant acid phosphatase; RANKL, receptor activator of nuclear factor-кB ligand; MMP-9, matrix metallopeptidase-9; CTSK, cathepsin K.

The data also revealed that bone resorptive pit areas increased with a higher concentration of RANKL (100 ng/ml; Fig. 3C). However, the bone resorption of osteoclasts induced by RANKL was markedly attenuated by exposure to $50 \mathrm{~Hz}$ PEMFs (Fig. 3B and D), which was consistent with the inhibitory effects of PEMFs on osteoclast formation, and in turn implied the active bone resorption activity of multinucleated osteoclasts. These results indicated that PEMF repressed the bone resorption activity of osteoclasts in vitro.

Effect of PEMF on osteoclastic differentiation. To further elucidate the role of PEMF in osteoclast differentiation, the present study examined the expression of osteoclastic marker genes during osteoclastogenesis using RT-qPCR. The expression of NFATc1, one of the osteoclast-specific transcription factors, as well as that of three other osteoclastic specific genes, MMP-9, TRAP and CTSK, was upregulated upon treatment with RANKL. However, this was significantly decreased by the addition of PEMF (Fig. 4A-D). This PEMF-regulated expression of osteoclastogenic markers were further confirmed by western blotting (Fig. 4E and F). Collectively, these data supported the inhibition of osteoclast formation by PEMFs.

PEMFs inhibit RANKL-induced osteoclastogenesis via regulation of the Akt/mTOR signaling pathway. To evaluate 
A

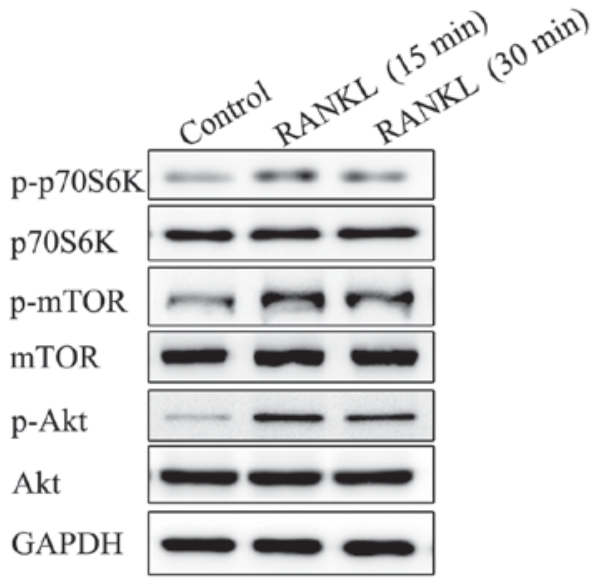

C

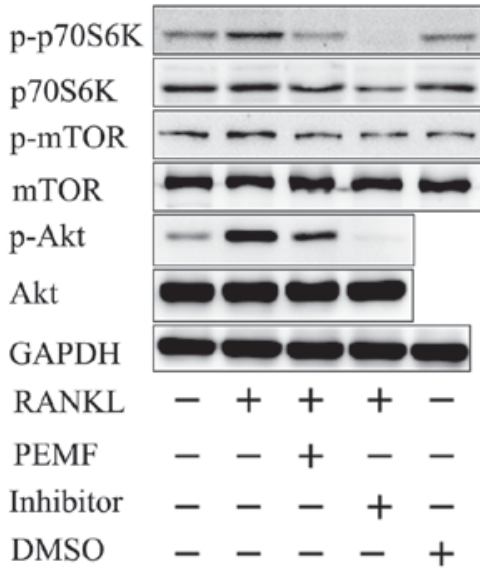

B

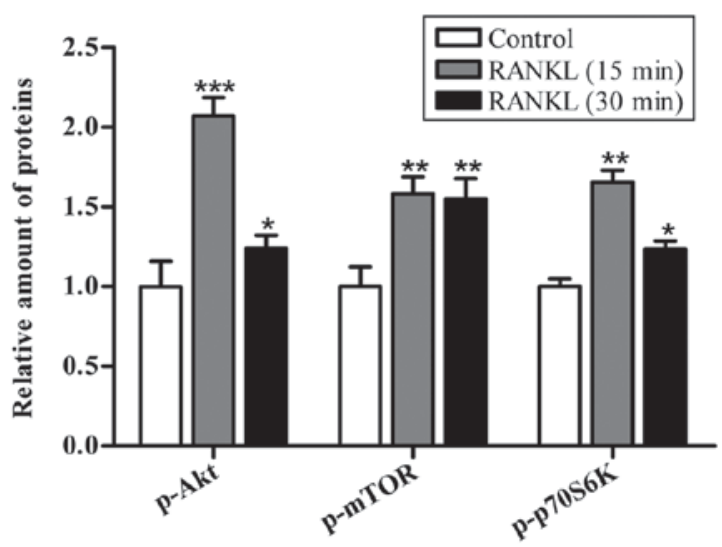

D

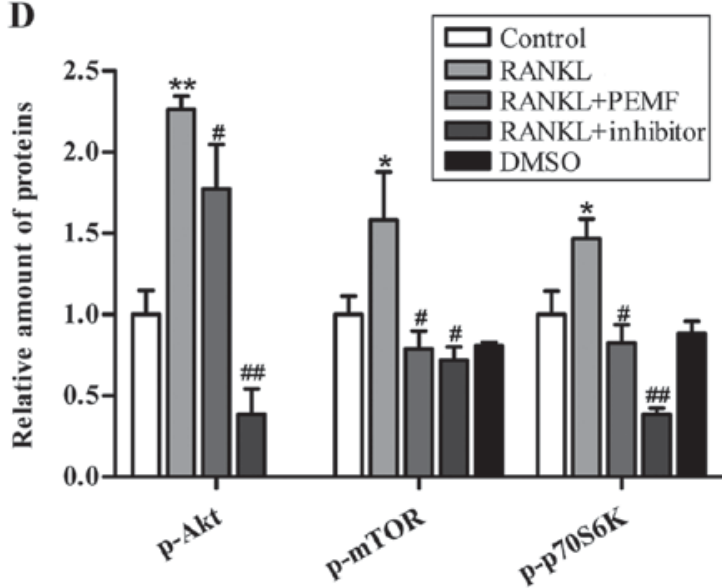

Figure 5. PEMF reduces RANKL-induced osteoclastogenesis via inhibition of the Akt/mTOR signaling pathway. (A) Following serum-starvation for $6 \mathrm{~h}$, RAW264.7 cells were stimulated with RANKL (50 ng/ml) for 15 or $30 \mathrm{~min}$. Cell lysates were prepared for immunoblotting with antibodies, as indicated. (B) Relative protein expression levels were measured using ImageJ software. GAPDH served as a loading control. (C) Following serum-starvation for $6 \mathrm{~h}$, RAW264.7 cells were incubated for 15 min with RANKL (50 ng/ml), RANKL (50 ng/ml) + PEMF or RANKL (50 ng/ml) with pharmacological inhibitors [perifosine (Akt inhibitor, $2.5 \mu \mathrm{M}$, dissolved in water) and rapamycin (mTOR and p70S6K inhibitor, $1 \mu \mathrm{M}$, dissolved in dimethyl sulfoxide)]. Total protein was extracted from each group and evaluated by western blotting. (D) Relative protein expression levels were measured using ImageJ software. GAPDH served as a loading control. Data are presented as the mean \pm standard deviation of three independent experiments. ${ }^{*} \mathrm{P}<0.05,{ }^{* *} \mathrm{P}<0.01,{ }^{* * *} \mathrm{P}<0.001$ vs. control group; ${ }^{\#} \mathrm{P}<0.05,{ }^{\# \#} \mathrm{P}<0.01$ vs. RANKL group, based on Student's t-tests and one-way analysis of variance. PEMF, pulsed electromagnetic fields; RANKL, receptor activator of nuclear factor-kB ligand; Akt, protein kinase B; mTOR, mammalian target of rapamycin.

the effect of PEMF on RANKL signaling activation following RANKL treatment in RAW264.7 cells, proteins associated with the Akt/mTOR signaling pathway, including Akt, mTOR and p70S6K, were examined by western blotting. As demonstrated in Fig. 5A, the data revealed that there was no significant difference among groups in the total protein expression levels of Akt, mTOR and p70S6K. However, the expression of p-Akt, p-mTOR and p-p70S6K, the type of modified protein with a crucial role in this signal transduction, was increased in response to RANKL treatment (Fig. 5B). Conversely, the upregulation of these proteins induced by RANKL was suppressed by pharmacological inhibitors, perifosine (Akt inhibitor) and rapamycin (mTOR and p70S6K inhibitor). In accordance with the inhibitory effects of antagonists, PEMF application also distinctly decreased RANKL-induced upregulation of these phosphorylated proteins (Fig. 5C and D), suggesting the possibility that PEMF may inhibit RANKL-induced osteoclastogenesis by suppression of the Akt/mTOR signaling pathway./

\section{Discussion}

Osteoclasts, the specialized cells derived from monocyte/ macrophage haematopoietic lineage, develop and adhere to bone matrix, and subsequently secrete acid and lyase that degrade it in an extracellular compartment (24). Due to excess osteoclastic activity, bone resorption is accelerated resulting in an imbalance of bone remodeling, leading to osteoporosis and other associated diseases, particularly in the elderly population (30). Osteoporosis is an important issue in orthopedics. PEMF has been successfully applied to various diseases in several basic research experiments, most commonly including senile osteoporosis, osteoarthritis and cancer bone metastasis. However, few studies have reported the effects of PEMF on osteoclast differentiation and formation, and the associated signaling pathway mechanisms $(31,32)$. A previous study demonstrated that PEMF exposure markedly inhibited RANKL-induced osteoclast formation from RAW264.7 macrophages, and that this effect 
resulted from suppression of intracellular Akt and mTOR protein phosphorylation.

RANKL and M-CSF are necessary for the differentiation and maturation of osteoclasts, which then activate the RANK receptor on the surface of haematopoietic precursor cells (33). Macrophage RAW264.7 cells are capable of secreting adequate M-CSF independently to support their osteoclast differentiation. This cell line is known to be suitable for investigating RANKL-induced osteoclast formation in vitro due to the high levels of RANK it produces (34). The present study demonstrated that RANKL led to a significant increase in the number of osteoclasts, and the mRNA and protein expression of osteoclastic markers throughout the 4 day experimental period. However, the marked increases in the number of multinucleated giant osteoclasts and the expression of osteoclastic-specific marker were suppressed by PEMF exposure during osteoclastogenesis. Furthermore, it was demonstrated that PEMFs inhibited RANKL-induced Akt and $\mathrm{mTOR}$ activation. Therefore, these results revealed that PEMF exposure reversed RANKL-induced osteoclast differentiation and formation via suppression of the Akt/mTOR signaling pathway.

RANKL, a tumor necrosis factor (TNF) family member, serves an important role in osteoclast survival, cytoskeletal reorganization, bone resorption and osteoclast differentiation. Activation of RANK by its ligand is crucial for osteoclastogenesis. Wong et al (35) demonstrated that RANKL activates the anti-apoptotic serine/threonine kinase Akt through a signaling complex involving TNF receptor-associated factor (TRAF) 6 and c-Src in osteoclasts. Akt, also known as protein kinase $\mathrm{B}$, has been demonstrated to be vital in osteoclast differentiation (36). Furthermore, Akt functions to phosphorylate mTOR, a protein involved in cell growth, homeostasis and cell differentiation via the phosphatidylinositol 3-kinase (PI3K)/Akt signaling pathway (37). Inhibition of mTOR by rapamycin, an mTOR inhibitor, decreases the number of multinucleated TRAP-positive osteoclasts in the chondro-osseous junction in rats (38). Following the activation of Akt and mTOR protein, the downstream signaling pathways are activated and osteoclast differentiation is promoted.

Despite the future potential of arising therapeutic strategies revealed by these studies, there are also limitations. The specific mechanism by which PEMF inhibits the Akt/mTOR signaling pathway remains unclear. One possibility is that PEMF influences the Src signaling pathway, which is a significant RANK signaling network in osteoclasts. Src, a signaling protein required for osteoclast activation, has also been revealed to bind to TRAF6 and allow RANK-mediated signaling to proceed through lipid kinase phosphatidylinositol $3 \mathrm{OH}$ kinase and serine/threonine protein kinase Akt (39). Src has an important role in the signal transduction of the RANK/RANKL/OPG signaling network. The activation or inhibition of Src signaling pathways may influence cell proliferation, differentiation, motility and cytoskeletal rearrangement (24). However, further investigation is required to elucidate the specific underlying mechanisms and associations.

In conclusion, the results of the present study demonstrated that PEMF $(50 \mathrm{~Hz}, 1 \mathrm{mT})$ inhibited osteoclast differentiation from RAW264.7 macrophages by inhibiting the Akt/mTOR signaling pathway. Therefore, PEMF may be an effective non-invasive method in the treatment of a wide range of osteoclast-associated diseases. Understanding the anti-osteoclastogenesis mechanism of PEMF may be helpful for the efficient use of PEMF in osteoporosis-associated diseases, both in animal experiments and as a future therapeutic strategy.

\section{Acknowledgements}

The authors gratefully acknowledge the technical assistance of Dr Hui Zhou and Mr Zhonghao Li of the Department of Pathophysiology, Key Laboratory for Shock and Microcirculation Research of Guangdong, Southern Medical University (Guangzhou, China). The authors would also like to thank the Department of Pathophysiology, Southern Medical University (Guangzhou, China) for allowing the use of the laboratory.

\section{Funding}

No funding was received.

\section{Availability of data and materials}

All data generated or analyzed during this study are included in this published article.

\section{Authors' contributions}

YL, JS, HX, QY, MZ, JT designed the experiments. YL performed the major experiments and data analysis. JS, HX performed a part of the experiments. YL drafted the manuscript. All authors read and approved the final version of the manuscript.

\section{Ethics approval and consent to participate}

Not applicable.

\section{Consent for publication}

Not applicable.

\section{Competing interests}

The authors declare that they have no competing interests.

\section{References}

1. Liu W, Yang LH, Kong XC, An LK and Wang R: Meta-analysis of osteoporosis: Fracture risks, medication and treatment. Minerva Med 106: 203-214, 2015.

2. Musette P, Brandi ML, Cacoub P, Kaufman JM, Rizzoli R and Reginster JY: Treatment of osteoporosis: Recognizing and managing cutaneous adverse reactions and drug-induced hypersensitivity. Osteoporos Int 21: 723-732, 2010.

3. Gartlehner G, Patel SV, Feltner C, Weber RP, Long R, Mullican K, Boland E, Lux L and Viswanathan M: Hormone therapy for the primary prevention of chronic conditions in postmenopausal women: Evidence report and systematic review for the US preventive services task force. JAMA 318: 2234-2249, 2017. 
4. Hadji P, Papaioannou N, Gielen E, Feudjo Tepie M, Zhang E, Frieling I, Geusens P, Makras P, Resch H, Möller G, et al: Persistence, adherence, and medication-taking behavior in women with postmenopausal osteoporosis receiving denosumab in routine practice in Germany, Austria, Greece and Belgium: 12-month results from a European non-interventional study. Osteoporosis Int 26: 2479-2489, 2015.

5. Bone HG, Dempster DW, Eisman JA, Greenspan SL, McClung MR, Nakamura T, Papapoulos S, Shih WJ, Rybak-Feiglin A, Santora AC, et al: Odanacatib for the treatment of postmenopausal osteoporosis: Development history and design and participant characteristics of LOFT, the long-term Odanacatib fracture trial. Osteoporosis Int 26: 2721, 2015.

6. Hannon RA, Clack G, Rimmer M, Swaisland A, Lockton JA, Finkelman RD and Eastell R: Effects of the Src kinase inhibitor saracatinib (AZD0530) on bone turnover in healthy men: A randomized, double-blind, placebo-controlled, multiple-ascending-dose phase I trial. J Bone Miner Res 25: 463-471, 2013.

7. Bonnick S, De Villiers T, Odio A, Palacios S, Chapurlat R DaSilva C, Scott BB, Le Bailly De Tilleghem C, Leung AT and Gurner D: Effects of odanacatib on BMD and safety in the treatment of osteoporosis in postmenopausal women previously treated with alendronate: A randomized placebo-controlled trial. J Clin Endocrinol Metab 98: 4727-4735, 2013.

8. Bassett CA, Pawluk RJ and Pilla AA: Augmentation of bone repair by inductively coupled electromagnetic fields. Science 184 575-577, 1974.

9. Bassett CA, Pawluk RJ and Pilla AA: Acceleration of fracture repair by electromagnetic fields. A surgically noninvasive method. Ann N Y Acad Sci 238: 242-262, 1974.

10. Wang T, Wang P, Cao Z, Wang X, Wang D, Shen Y, Jing D, Luo E and Tang W: Effects of BMP9 and pulsed electromagnetic fields on the proliferation and osteogenic differentiation of human periodontal ligament stem cells. Bioelectromagnetics 38: 63-77, 2017.

11. Zhou J, He H, Yang L, Chen S, Guo H, Xia L, Liu H, Qin Y, Liu C, Wei X, et al: Effects of pulsed electromagnetic fields on bone mass and Wnt/ $\beta$-catenin signaling pathway in ovariectomized rats. Arch Med Res 274-282, 2012.

12. Jing D, Zhai M, Tong S, Xu F, Cai J, Shen G, Wu Y, Li X, Xie K, Liu J, et al: Pulsed electromagnetic fields promote osteogenesis and osseointegration of porous titanium implants in bone defect repair through a $W n t / \beta$-catenin signaling-associated mechanism Sci Rep 24: 32045, 2016.

13. Urnukhsaikhan E, Cho H, Mishig-Ochir T, Seo YK and Park JK Pulsed electromagnetic fields promote survival and neuronal differentiation of human BM-MSCs. Life Sci 151: 130-138, 2016.

14. Kang KS, Hong JM, Seol YJ, Rhie JW, Jeong YH and Cho DW: Short-term evaluation of electromagnetic field pretreatment of adipose-derived stem cells to improve bone healing. J Tissue Eng Regen Med 9: 1161-1171, 2012

15. He J, Zhang Y, Chen J, Zheng S, Huang H and Dong X: Effects of pulsed electromagnetic fields on the expression of NFATc1 and CAI in mouse osteoclast-like cells. Aging Clin Exp Res 27: 13-19, 2015.

16. Lei T, Liang Z, Li F, Tang C, Xie K, Wang P, Dong X, Shan S, Jiang M, Xu Q, et al: Pulsed electromagnetic fields (PEMF) attenuate changes in vertebral bone mass, architecture and strength in ovariectomized mice. Bone 108: 10-19, 2017.

17. Lin $\mathrm{HY}$ and Lin YJ: In vitro effects of low frequency electromagnetic fields on osteoblast proliferation and maturation in an inflammatory environment. Bioelectromagnetics 32: 552-560, 2011

18. Zhang D, Huang Y, Huang Z, Zhang R, Wang H and Huang D: FTY-720P suppresses osteoclast formation by regulating expression of interleukin-6 (IL-6), interleukin-4 (IL-4) and matrix metalloproteinase 2 (MMP-2). Med Sci Monitor 22: 2187-2194, 2016.

19. Yang J, Zhang J, Ding C, Dong D and Shang P: Regulation of osteoblast differentiation and iron content in MC3T3-E1 cells by static magnetic field with different intensities. Biol Trace Elem Res: Oct 19,2017 (Epub ahead of print). doi: 10.1007/s12011-017-1161-5.

20. Barnaba SA, Ruzzini L, Di Martino A, Lanotte A, Sgambato A and Denaro V: Clinical significance of different effects of static and pulsed electromagnetic fields on human osteoclast cultures. Rheumatol Int 32: 1025-1031, 2012.

21. Xie YF, Shi WG, Zhou J, Gao YH, Li SF, Fang QQ, Wang MG, Ma HP, Wang JF, Xian CJ, et al: Pulsed electromagnetic fields stimulate osteogenic differentiation and maturation of osteoblasts by upregulating the expression of BMPRII localized at the base of primary cilium. Bone 93: 22-32, 2016.
22. Jing D, Li F, Jiang M, Cai J, Wu Y, Xie K, Wu X, Tang C, Liu J, Guo W, et al: Pulsed electromagnetic fields improve bone microstructure and strength in ovariectomized rats through a Wnt/Lrp5/ $\beta$-catenin signaling-associated mechanism. PLoS One 8: e79377, 2013.

23. Chang K, Chang WH, Huang S, Huang S and Shih C: Pulsed electromagnetic fields stimulation affects osteoclast formation by modulation of osteoprotegerin, RANK ligand and macrophage colony-stimulating factor. J Orthop Res 23: 1308-1314, 2005.

24. Boyle WJ, Simonet WS and Lacey DL: Osteoclast differentiation and activation. Nature 423: 337-342, 2003

25. Xu H, Zhang J, Lei Y, Han Z, Rong D, Yu Q, Zhao M and Tian J: Low frequency pulsed electromagnetic field promotes $\mathrm{C} 2 \mathrm{C} 12$ myoblasts proliferation via activation of MAPK/ERK pathway. Biochem Biophys Res Commun 479: 97-102, 2016.

26. Park KH, Park B, Yoon DS, Kwon SH, Shin DM, Lee JW, Lee HG, Shim JH, Park JH and Lee JM: Zinc inhibits osteoclast differentiation by suppression of $\mathrm{Ca}^{2+}$-Calcineurin-NFATc1 signaling pathway. Cell Commun Signal 11: 74, 2013.

27. Kapetanakis NI, Uzan C, Jimenez-Pailhes AS, Gouy S, Bentivegna E, Morice P, Caron O, Gourzones-Dmitriev C, Le Teuff G and Busson P: Plasma miR-200b in ovarian carcinoma patients: Distinct pattern of pre/post-treatment variation compared to CA-125 and potential for prediction of progression-free survival. Oncotarget 6: 36815-36824, 2015.

28. Halleen JM, Tiitinen SL, Ylipahkala H, Fagerlund KM and Väänänen HK: Tartrate-resistant acid phosphatase $5 \mathrm{~b}$ (TRACP 5b) as a marker of bone resorption. Clin Lab 52: 499-509, 2006.

29. Liu H, Li D, Liu S, Liu Z and Li M: Histochemical evidence of IGF2 mRNA-binding protein 2-mediated regulation of osteoclast function and adhesive ability. Histochem Cell Biol 149: 343-351, 2018.

30. Rachner TD, Khosla S and Hofbauer LC: Osteoporosis: Now and the future. Lancet 377: 1276-1287, 2011.

31. Liu HF, He HC, Yang L, Yang ZY, Yao K, Wu YC, Yang XB and He CQ: Pulsed electromagnetic fields for postmenopausal osteoporosis and concomitant lumbar osteoarthritis in southwest China using proximal femur bone mineral density as the primary endpoint: Study protocol for a randomized controlled trial. Trials 16: 265, 2015.

32. Muramatsu Y, Matsui T, Deie M and Sato K: Pulsed electromagnetic field stimulation promotes anti-cell proliferative activity in doxorubicin-treated mouse osteosarcoma cells. In Vivo 31: 61-68, 2017.

33. Visagie A, Kasonga A, Deepak V, Moosa S, Marais S, Kruger MC and Coetzee M: Commercial Honeybush (cyclopia spp.) tea extract inhibits osteoclast formation and bone resorption in RAW264.7 murine macrophages-an in vitro study. Int J Environ Res Public Health 12: 13779-13793, 2015.

34. Collin-Osdoby P and Osdoby P: RANKL-mediated osteoclast formation from murine RAW 264.7 cells. Methods Mol Biol 816: 187-202, 2012.

35. Wong BR, Besser D, Kim N, Arron JR, Vologodskaia M, Hanafusa $\mathrm{H}$ and Choi Y: TRANCE, a TNF family member, activates Akt/PKB through a signaling complex involving TRAF6 and c-Src. Mol Cell 4: 1041-1049, 1999.

36. Li L, Sapkota M, Gao M, Choi H and Soh Y: Macrolactin F inhibits RANKL-mediated osteoclastogenesis by suppressing Akt, MAPK and NFATc1 pathways and promotes osteoblastogenesis through a BMP-2/smad/Akt/Runx2 signaling pathway. Eur J Pharmacol 815: 202-209, 2017.

37. Navé BT, Ouwens M, Withers DJ, Alessi DR and Shepherd PR: Mammalian target of rapamycin is a direct target for protein kinase B: Identification of a convergence point for opposing effects of insulin and amino-acid deficiency on protein translation. Biochem J 344: 427-431, 1999.

38. Hsieh CJ, Kuo PL, Hou MF, Hung JY, Chang FR, Hsu YC, Huang YF, Tsai EM and Hsu YL: Wedelolactone inhibits breast cancer-induced osteoclastogenesis by decreasing Akt/mTOR signaling. Int J Oncol 46: 555-562, 2015

39. Takeshita S: SHIP-deficient mice are severely osteoporotic due to increased numbers of hyperresorptive osteoclasts. Nat Med 9: 943-949, 2002. 